\title{
On seasonal error correction when the processes include different numbers of unit roots
}

\author{
Johan Lyhagen and Mårten Löf* \\ SSE/EFI Working Paper Series in Economics and Finance, No. 0418
}

February 13, 2001

\begin{abstract}
We propose a seasonal cointegration model [SECM] for quarterly data which includes variables with different numbers of unit roots and thus needs to be transformed in different ways in order to yield stationarity. A Monte Carlo simulation is carried out to investigate the consequences of specifying a SECM with all variables in annual diffrerences in this situation. The SECM in annual differences is compared to the correctly specified model. Pre-testing for unit roots using two different approaches, and where the models are specified according to the unit root test results, is also considered. The forecast mean squared error criterion and certain parameter estimation results indicate that, in practice, a cointegration model where all variables are transformed with the annual difference filter is more robust than one obtained by pre-testing for a smaller number of unit roots. The second best choice when the true model is not known and when the aim is to forecast, is an ordinary VAR model also in annual differences.
\end{abstract}

Keywords: Seasonal cointegration; forecasting

JEL: C32; C53

${ }^{*}$ Department of Economic Statistics, Stockholm School of Economics, P.O Box 650111383 Stockholm, E-mail: stjl@hhs.se and stmlo@hhs.se. 


\section{Introduction}

Cointegration was a major break through when introduced by Engle and Granger (1987). They treat cointegration as stable long-run relationships between a set of nonstationary time series processes, which includes unit roots at the nonseasonal, or zero, frequency. One of the main attractions of the idea is that the cointegrating vectors may be interpreted as equilibrium relations between the variables. It is often assumed that the seasonal patterns are constant over time and many applied researchers try to model these variations using deterministic seasonal dummies. However, substantial empirical evidence makes it safe to conclude that the seasonal pattern in many economic time series is far from constant. To the best of our knowledge, there are two major routes to cointegration in the case of changing seasonal variation, namely periodic and seasonal cointegration. Periodic cointegration models, see for example Boswijk and Franses (1995), consider long-run relationships season by season, whereas seasonal cointegration models are based on the idea of the unit roots (zero and seasonal) implied by the annual difference filter, see Hylleberg et al. (1992) [HEGY]. In the present paper the latter model class for quarterly data is considered, a class to which Engle et al. (1993) [EGHL], Lee (1992) and Johansen and Schaumburg (1998) have made important contributions. EGHL propose a two-step approach to test for the presence of seasonal and nonseasonal cointegration relationships whereas Lee (1992) suggests a multiple equation seasonal error correction model [SECM], which extends the maximum likelihood approach to the nonseasonal case, summarized in Johansen (1995). Finally, Johansen and Schaumburg (1998) refine the asymptotic theory for SECM and propose a general estimation procedure for the parameters corresponding to the annual frequency. The specification of the SECM is straightforward if all the included variables contain roots at the same, but not necessarily at all, frequencies.

One purpose of this paper is to show how the more general SECM, proposed by Johansen an Schaumburg (1998), could be specified in the case where the quarterly observed variables contain different numbers of unit roots, which is a common situation when working with real world data. We assume that the interest of an empirical study is a) to test for the number of cointegrating vectors and estimate these at the nonseasonal and seasonal frequencies, and b) to forecast. A Monte Carlo simulation is carried out to investigate the consequences of specifying a SECM which assumes four unit roots in each process and where the variables are transformed to yield stationarity accordingly, i.e. applying the annual difference filter. This specifica- 
tion is compared to the correctly specified model,attaching a different filter to each variable. Furthermore, we consider pre-testing for the number of seasonal unit roots in the univariate time series and specify models suggested by these tests. The two seasonal unit root tests are the familiar HEGY test and the seasonal KPSS [SKPSS] tests of Lyhagen (2000), respectively. It has been shown that the HEGY test has poor power against alternatives close to the null hypothesis. This implies that one may find evidence of too many roots, in practice, using this method. It is therefore interesting to include the SKPSS, which has the null hypothesis of no unit roots. In the forecasting exercise we also include a VAR model in annual differences.

The outline of the paper is as follows. Section 2 describes cointegration and seasonal cointegration, while Section 3 discusses the model for variables with different numbers of seasonal unit roots. The Monte Carlo setup is given in Section 4 and the results are analyzed in Section 5. Some conclusions end the paper.

\section{Cointegration and seasonal cointegration}

Let $L$ be the lag operator, i.e. $L^{d} Y_{t}=Y_{t-d}$ and define the first difference filter as $\Delta=(1-L)$. Furthermore, let $\Delta_{d}=\left(1-L^{d}\right)$. Consider a quarterly observed $p$ dimensional autoregressive process $Y_{t}$. The vector error correction model [VECM] can now be written as:

$$
\Delta Y_{t}=\Pi Y_{t-1}+\Gamma_{1} \Delta Y_{t-1}+\ldots+\Gamma_{p} \Delta Y_{t-p}+\Phi D_{t}+\varepsilon_{t},
$$

where $\Pi=\alpha \beta^{\prime}$ is of reduced rank $r$ and where $\Gamma_{1}, \ldots, \Gamma_{p}$ are lag matrices, see Johansen (1995). The deterministic terms may contain a constant and seasonal dummies. One crucial assumptions is that the first difference filter should remove all unit roots in the process that generates $Y_{t}$. However, economic time series which possess a changing seasonal pattern over time often include seasonal unit roots, in addition to the zero frequency root. If $\Delta_{4}$ filters, henceforth annual difference filters, are required to transform the vector $Y_{t}$ to yield stationarity, the time series are said to be seasonally integrated i.e. $Y_{t} \sim \mathrm{SI}(1)$. This filter assumes four unit roots, all of which lie on the unit circle. This can be seen from the following factorization:

$$
\Delta_{4}=\left(1-L^{4}\right)=(1-L)(1+L)(1+i L)(1-i L)
$$

where $i=\sqrt{-1}$. Now, the $(1-L)$ part correspond to the zero frequency or nonseasonal unit root, so the first difference filter only removes one of the roots. The 
$(1+L)(1+i L)(1-i L)$ part corresponds to the three seasonal unit roots, namely -1 and $\pm i$. The -1 root is often called the biannual root while the two complex conjugate roots, $\pm i$, are called the annual frequency roots. Johansen and Schaumburg (1998), henceforth JS, show that the following transformed processes are needed in the SECM, when the variables in $Y_{t}$ includes the roots at \pm 1 and $\pm i$ :

$$
\begin{aligned}
& Z_{1 t}=\frac{(1+L)(1+i L)(1-i L) L}{4} Y_{t} \\
& Z_{2 t}=-\frac{(1-L)(1+i L)(1-i L) L}{4} Y_{t} \\
& Z_{3 t}=\frac{(1-L)(1+L)(1-i L) L}{4 i} Y_{t} \\
& Z_{4 t}=-\frac{(1-L)(1+L)(1+i L) L}{4 i} Y_{t}
\end{aligned}
$$

The above filters can be found in the first rows of $Z_{1 t}, Z_{2 t}, Z_{3 t}$ and $Z_{4 t}$, respectively in the Appendix. Furthermore, $Z_{m t}$ for $m=1, \ldots, 4$ are asymptotically pairwise uncorrelated:

$$
T^{-2} \sum_{t=1}^{T} Z_{i t} Z_{j t}^{\prime} \stackrel{P}{\rightarrow} 0, i \neq j
$$

implying that the cointegration vectors and adjustment coefficients can be found by removing the reduced rank restriction on the other frequencies by concentrating out the associated regressors in a regression. JS propose the following SECM:

$$
\Delta_{4} Y_{t}=\sum_{i=1}^{2} \alpha_{i} \beta_{i}^{\prime} Z_{i t}+\alpha_{3} \beta_{3}^{*} Z_{3 t}+\alpha_{4} \beta_{4}^{*} Z_{4 t}+\sum_{j=1}^{p} \Gamma_{j} \Delta_{4} Y_{t-j}+\varepsilon_{t}
$$

where $\alpha_{3}=\alpha_{R}+i \alpha_{I}, \alpha_{4}=\alpha_{R}-i \alpha_{I}, \beta_{3}=\beta_{R}+i \beta_{I}$ and $\beta_{4}=\beta_{R}-i \beta_{I}$. Here $\beta_{i}^{*}$ denotes the complex conjugate of $\beta_{i}$. The Model above can now be rewritten in a form that contains only real terms, if one lets $Z_{3 t}=Z_{R t}+i Z_{I t}$ and $Z_{4 t}=Z_{R t}-i Z_{I t}$. The annual frequency part of, i.e. $\alpha_{3} \beta_{3}^{*} Z_{3 t}+\alpha_{4} \beta_{4}^{*} Z_{4 t}$, can now be written:

$$
\begin{aligned}
& \left(\alpha_{R}+i \alpha_{I}\right)\left(\beta_{R}-i \beta_{I}\right)^{\prime}\left(Z_{R t}+i Z_{I t}\right)+\left(\alpha_{R}-i \alpha_{I}\right)\left(\beta_{R}+i \beta_{I}\right)^{\prime}\left(Z_{R t}-i Z_{I t}\right) \\
= & 2\left(\alpha_{R} \beta_{R}^{\prime}+\alpha_{I} \beta_{I}^{\prime}\right) Z_{R t}+2\left(\alpha_{R} \beta_{I}^{\prime}-\alpha_{I} \beta_{R}^{\prime}\right) Z_{I t} .
\end{aligned}
$$


Note again that this is the appropriate specification if all roots are assumed to be present. It can be shown that the filters for the annual frequency equal:

$$
\begin{aligned}
Z_{R t} & =-\frac{1}{4}\left(L^{2}-L^{4}\right) Y_{t} \\
Z_{I t} & =-\frac{1}{4}\left(L-L^{3}\right) Y_{t}
\end{aligned}
$$

The asymptotic properties of the estimators and the LR-test for the number of cointegrating vectors for the different frequencies are given in JS. Tests for reduced rank $(r)$ at the zero and biannual frequencies can be performed using the trace statistic:

$$
-2 \log (H(r) \mid H(p))-T \sum_{i=r+1}^{P} \log \left(1-\widehat{\lambda}_{i}\right),
$$

where $H(r)$ is the null hypothesis and $H(p)$ is the alternative hypothesis of full rank. The eigenvalues, $1>\widehat{\lambda}_{1}>\ldots>\widehat{\lambda}_{p}$, which maximize the likelihood function, are obtained by solving eigenvalue problems based on residual vectors. The null hypothesis for this test is that there are at most $r$ cointegrating vectors. Inference on the reduced rank matrix $\Pi_{2}=\alpha_{2} \beta_{2}^{\prime}$ follows the usual non-seasonal case, i.e. for $\Pi$ in (1), see Johansen (1995). Tests for the number of cointegrating vectors are similar at the annual frequency. However, it should be noted that estimation of the cointegrating vectors at this frequency is not that straightforward and includes a rather involved iterative procedure. Here we use a switching algorithm suggested in JS, which provides estimators for $\beta_{R}$ and $\beta_{I}$ that are asymptotically equivalent to the maximum likelihood estimators, see also Löf and Lyhagen (1999).

\section{Different orders of integration}

In this section we study variables appearing in the same model, but containing different numbers of unit roots. Let $z_{1}, \ldots, z_{s}$ be complex numbers and let $z_{m}$ be a root of $|A(z)|=0$, where $A(z)$ is the characteristic polynomial of (1), expressed in levels. We want to present the model in error correction form, although the number of unit roots varies among the included variables. No series should be overdifferenced or be nonstationary at any frequency. This is achieved by relying on the following results in JS. Let: 


$$
\begin{aligned}
p(z) & =\prod_{m=1}^{s}\left(1-\bar{z}_{m} z\right) \\
p_{a}(z) & =\prod_{m \in a}\left(1-\bar{z}_{m} z\right)=\frac{p(z)}{\prod_{m \notin a}\left(1-\bar{z}_{m} z\right)}, \quad z \notin a .
\end{aligned}
$$

where $\bar{z}_{m}=z_{m}^{-1}$. If $a_{i}$ denotes the set of unit roots for variable $i$ then $p_{a_{i}}(z)$ cancels them. For example, if variable 1 includes unit roots at the nonseasonal and the biannual frequencies then:

$$
\begin{aligned}
p(z) & =\left(1-\frac{z}{1}\right)\left(1-\frac{z}{-1}\right)\left(1-\frac{z}{i}\right)\left(1-\frac{z}{-i}\right)=1-z^{4} \\
p_{a_{1}}(z) & =\frac{\left(1-\frac{z}{1}\right)\left(1-\frac{z}{-1}\right)\left(1-\frac{z}{i}\right)\left(1-\frac{z}{-i}\right)}{\left(1-\frac{z}{i}\right)\left(1-\frac{z}{-i}\right)}=(1-z)(1+z)
\end{aligned}
$$

Let $P(z)$ be a diagonal matrix with diagonal elements such that the element in entry $(i, i)$ cancels the roots of the $i$ th variable, i.e.

$$
P(z)=\left[\begin{array}{cccc}
p_{a_{1}}(z) & 0 & \cdots & 0 \\
0 & p_{a_{2}}(z) & \cdots & 0 \\
\vdots & \vdots & & \vdots \\
0 & 0 & \cdots & p_{a_{p}}(z)
\end{array}\right]
$$

It is shown in JS that the error correction formulation is a consequence of a Lagrange expansion of $A(z)$ around the points $z=0, z_{1}, z_{2}, \ldots, z_{s}$ as follows

$$
A(z)=P(z)+\sum_{m=1}^{s} A\left(z_{m}\right) \frac{P_{m}(z) z}{P_{m}\left(z_{m}\right) z_{m}}+P(z) z A_{0}(z)
$$

where $P_{m}(z)$ is the matrix consisting of $p_{a_{i}}(z) /\left(1-\bar{z}_{m} z\right)$, which is zero if $\left(1-\bar{z}_{m} z\right)$ does not belong to $p_{a_{i}}(z)$. If we again consider variable 1 and let $m=1$ (zero frequency), then $z_{m}=z_{1}=1$ and:

$$
\begin{aligned}
& \frac{p_{a_{1}}(z)}{\left(1-\bar{z}_{1} z\right)}=\frac{(1-z)(1+z)}{\left(1-\frac{z}{1}\right)}=1+z \\
& \frac{P_{1}(z) z}{P_{1}\left(z_{1}\right) z_{1}}=\frac{(1+z) z}{(1+1) 1}=\frac{(1+z) z}{2}
\end{aligned}
$$


The proof of (10) is a generalization of the one in JS. Each entry of

$$
A(z)-P(z)-\sum_{m=1}^{s} A\left(z_{m}\right) \frac{P_{m}(z) z}{P_{m}\left(z_{m}\right) z_{m}}
$$

is zero for $z=0, z_{1}, z_{2}, \ldots, z_{s}$ Hence, the difference can be written as $P(z) z A_{0}(z)$ for some matrix polynomial $A_{0}(z)$. Another consequence is the following. Let $z_{1}$, $z_{2}, \ldots, z_{s}$ be the unit roots of $|A(z)|=0$, such that the matrices $A\left(z_{m}\right)$ are of reduced rank. Then $Y_{t}$ has an error correction representation

$$
\begin{aligned}
P(L) Y_{t} & =\sum_{m=1}^{s} \alpha_{m} \beta_{m}^{*} \frac{P_{m}(L) L}{P_{m}\left(z_{m}\right) z_{m}} Y_{t}-P(L) A_{0}(L) L Y_{t}+\varepsilon_{t} \\
& =\sum_{m=1}^{s} \alpha_{m} \beta_{m}^{*} Z_{m t}-P(L) A_{0}(L) L Y_{t}+\varepsilon_{t} .
\end{aligned}
$$

The filters $Z_{m t}$ in (12) equal those of (3) if the processes includes the roots \pm 1 and $\pm i$. As an example, consider a situation where the variables may have the roots at the nonseasonal and at the biannual frequency. Let $Y_{t}$ be a $T \times p$ vector, where the variables are ordered such that only the last $k$ variables include both roots. That is, let $Y_{t}=\left[Y_{1 t}, Y_{2 t}\right]^{\prime}$ where $Y_{1 t}$ includes the nonseasonal, or zero, frequency root, while the $Y_{2 t}$ variables includes both roots. The most important matrices in (12) are:

$$
\begin{aligned}
P(L) & =\left[\begin{array}{cc}
(1-L) & 0 \\
0 & (1-L)(1+L)
\end{array}\right] \\
\frac{P_{1}(L) L}{P_{1}(1) 1} & =\left[\begin{array}{cc}
L & 0 \\
0 & \frac{(1+L) L}{2}
\end{array}\right] \\
\frac{P_{2}(L) L}{P_{2}(-1)-1} & =\left[\begin{array}{cc}
0 & 0 \\
0 & -\frac{(1-L) L}{2}
\end{array}\right]
\end{aligned}
$$

Using these, it is easily shown that the error correction model for $Y_{t}$ is 


$$
\begin{aligned}
& {\left[\begin{array}{cc}
(1-L) & 0 \\
0 & (1-L)(1+L)
\end{array}\right]\left[\begin{array}{c}
Y_{1 t} \\
Y_{2 t}
\end{array}\right] } \\
= & \alpha_{1} \beta_{1}^{\prime}\left[\begin{array}{cc}
L & 0 \\
0 & \frac{(1+L) L}{2}
\end{array}\right]\left[\begin{array}{c}
Y_{1 t} \\
Y_{2 t}
\end{array}\right] \\
- & \alpha_{2} \beta_{2}^{\prime}\left[\begin{array}{cc}
0 & 0 \\
0 & \frac{(1-L) L}{2}
\end{array}\right]\left[\begin{array}{c}
Y_{1 t} \\
Y_{2 t}
\end{array}\right]+\varepsilon_{t}
\end{aligned}
$$

The Appendix presents the appropriate transformations of the variables in this situation. These can be found in the second and last rows of the $P(L)$ matrix, respectively. Moreover, the filtered variables, used for the zero frequency in (14), can be found in the second and last rows of $Z_{1 t}$, respectively. Finally, the appropriate filters, for the variables in $Y_{2 t}$, at the biannual frequency can be found in the second row of $Z_{2 t}$. One implication is that the $k$ last variables, having the roots 1 and -1 , can not be just a single variable. That is, one must require $k>1$ to be able test for cointegration at the biannual frequency.

Modeling is usually done in steps, starting from pre-testing for seasonal unit roots, using e.g. the HEGY-test. Cointegration is then tested using the corresponding adjusted model. A problem with the HEGY approach is that, asymptotically, there is a chance, $\varepsilon$, of rejecting a true null of a unit root, where $\varepsilon$ is the chosen significance level. Hence, the method is not asymptotically efficient. A test which has the null of no unit root, proposed by Lyhagen (2000), may be a better choice because it asymptotically rejects a false null and a tendency to accept more rather than less unit roots may lead to more robust models. It has also been shown that the HEGY test has low power against alternatives, close to the null hypothesis. This implies that one tends to find evidence of nonstationarity when the process is in fact stationary at a certain frequency. So, when considering small samples, it is an open question weather one should use the HEGY approach or SKPSS. 


\section{Monte Carlo}

Seven bivariate DGPs are considered, where the first variable $y_{1 t}$ always includes all unit roots. The set of true unit roots of $y_{2 t}$ is different in each DGP:

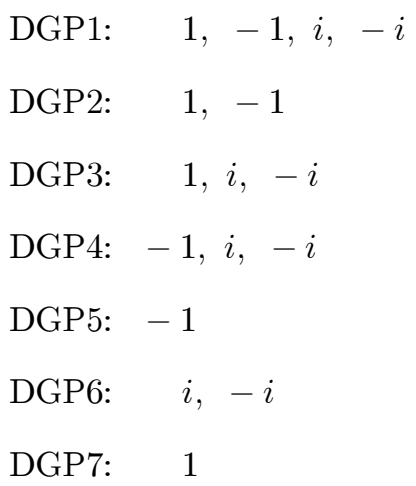

Cointegration prevails if $y_{2 t}$ is nonstationary at a certain frequency, because $y_{1 t}$ includes all possible unit roots. For example, cointegration can apply at all frequencies if DGP1 is considered. On the other hand, cointegration is only possible at the zero and biannual frequency if DGP2 is used. We consider sample sizes of $T=40,80$ and 120 . The number of observations is close to the ones often found in empirical applications. The number of replicates is 10000 . The significance level is $5 \%$ throughout. The seven DGPs are based on estimates on income and consumption data for Japan (in logs), previously used in EGHL. To achieve well behaved DGPs, a constant and a time trend are before estimation extracted from the variables. All the DGPs have eigenvalues of the companion matrix either on or inside the unit circle, see Table 1.

Four model specifications are compared when cointegration is considered. In the forecasting exercise we include a fifth model (Model 5) which is a vector autoregressive model $[\mathrm{VAR}]$ in annual differences. When Models 2 and 5 are used, all variables are transformed using the annual difference directly. In Model 1 we use the annual difference filter for $y_{1 t}$ and difference $y_{2 t}$ according to the true number of unit roots, see $P(L)$ in the Appendix for the seven DGP cases. Finally, in Models 3 and 4 we apply the annual difference filter for $y_{1 t}$, without pre-testing for the number of unit roots, while $y_{2 t}$ is differenced according to the unit root test results. We summarize 
the different models below:

Table 4.1: Models used in the Monte Carlo study.

\begin{tabular}{ccccc}
\hline \hline & & & \multicolumn{2}{c}{ Pre-testing } \\
\hline Model & & Cointegration & $y_{1 t}$ & $y_{2 t}$ \\
\hline 1 & True SECM & Yes & No & No \\
2 & $\Delta_{4}$ SECM & Yes & No & No \\
3 & HEGY $\Rightarrow$ SECM & Yes & No & Yes \\
4 & SKPSS $\Rightarrow$ SECM & Yes & No & Yes \\
5 & $\Delta_{4}$ VAR & No & No & No \\
\hline \hline
\end{tabular}

For Models 1 to 4, tests for rank zero against full rank (rank two) and tests for rank one against full rank are considered at all frequencies. Size and power properties are evaluated by estimating the proportion of rejections for each frequency. To evaluate the estimates of the parameters in position $i, j$ of the $\Pi$ matrices we use the mean of the mean squared error [MSE]:

$$
\mathrm{MSE}=\sum_{r=1}^{R} \frac{\sum_{i} \sum_{j}\left(\hat{\Pi}_{i j}-\Pi_{i j}\right)^{2} / 4}{R}
$$

where $R$ equals the number of replicates. We also forecast 12 periods ahead to compare the model specifications. As noted above we include Model 5 in this case. Forecasting accuracy is measured using the determinant $\left(\operatorname{Det}_{k}\right)$ and the trace $\left(\operatorname{Tr}_{k}\right)$ of the mean squared error matrix, respectively:

$$
\begin{aligned}
\operatorname{Det}_{k} & =\left|\sum_{r=1}^{R} \frac{\left(x_{t+k}-f_{t+k}\right)^{\prime}\left(x_{t+k}-f_{t+k}\right)}{R}\right| \\
\operatorname{Tr}_{k} & =\operatorname{trace} \sum_{r=1}^{R} \frac{\left[\left(x_{t+k}-f_{t+k}\right)^{\prime}\left(x_{t+k}-f_{t+k}\right)\right]}{R}
\end{aligned}
$$

where again $R$ equals the number of replicates.

\section{Monte Carlo results}

The results for size and power are displayed in Tables 2 to 7 and the MSE of the parameter estimates in Tables 8 to 11. We also show a parts of the forecasting exercise in Table 12. The first thing to note is that the model which is transformed according to the true number of roots generally performs better than the other 
models, concerning inference as well as estimation and forecasting.

Its closest competitor, if one wants to reject a false null of $r=0$, is the model which assumes the presence of all roots (Model 2), see for example the columns of DGP2 and DGP3 in Table 2 except for $T=40$. Note the results for DGP2 and DGP4 in Table 3 for the biannual frequency tests or DGP3, DGP4 and DGP6 in Table 4 if the annual frequency is considered. Model 2 is also likely to reject a true null hypothesis of $r=0$ in some cases. One example is DGP4, which includes the roots -1 and $\pm i$, and when the test concern cointegration at the zero frequency, see Table 2. Another example is DGP6, which only includes the two complex roots, when the biannual frequency is considered, see Table 3. Similar results for Model 2 can be found when DGP2, DGP5 and DGP7 are used an when the tests concern the annual frequency. These results are due to the fact that $y_{2 t}$ constitutes a stationary 'relation' by itself in these cases. So, it is quite logical that the null hypothesis of $r=0$ is rejected. Note that cointegration should not apply, since $y_{1 t}$ is a nonstationary variable. These cases are underlined in Tables 2 to 4 and in Tables 8 to 11 .

SKPSS (Model 4) is to prefer in some cases, whereas pre-testing with HEGY (Model 3) seems to be a better strategy in other cases. For example, HEGY fails if DGP7 is used and if the test concerns zero frequency cointegration (Table 2), whereas SKPSS fails if DGP1 is used and the objective is to test for cointegration at the biannual frequency (Table 3). SKPSS is slightly better in the cases when the root for which cointegrating is tested for is not present in $y_{2 t}$, while HEGY seems to have better power when there in fact should be cointegration.

The size for Model 1 and 2, when testing the true null hypothesis of $r=1$, tends to the nominal with sample size and is fairly close to the nominal. Pre-testing with SKPSS seems to work except in DGP1 where it is greatly oversized (zero and annual frequencies, Tables 5 and 7) or very undersized (biannual frequency, Table 6). The size when pre-testing with HEGY is diverging from the nominal for DGP2 and DGP7 at the zero frequency, while it works better for other DGPs, see Table 5. Similar results can be found in the columns for DGP2, DGP4 and DGP5 if the biannual frequency is considered, see Table 6 , but also at the annual frequency in some cases.

For Models 1 and 2 the MSE of the parameter estimates decreases uniformly with sample size. This is also true for SKPSS, except for $\Pi_{3}$ when using DGP1, see Table 10. Pre-testing with the HEGY, on the other hand, either increases MSE with sample size or slowly decreases it. The use of Model 2 sometimes results in 
much higher MSE as compared to the other methods, see for example the MSE for $\Pi_{2}$ when DGP3 and DGP6 are considered in Table 9. This last result depends on the estimation of a nonzero rank matrix, where $y_{2 t}$ is stationary, see discussion above.

The results of the forecasting exercise are almost always the same, regardless if the determinant or the trace of the MSE matrix is used. In Table 12 we present the ranking of the models after averaging over the 12 forecast periods, and the measure used is the determinant of the MSE matrix. The ranking is quite stable over sample sizes and DGPs. The model that allows for all unit roots (Model 2) comes as a good second after the true model (Model 1) in most cases. Model 5 i.e. the VAR model in annual differences seems to be a better choice than using models resulting from the two pre-testing methods in most cases. Model 5 has actually a better performance than Model 2 in some cases when $T=40$. This is most likely due to the parameter uncertainty when estimating Model 2 in small samples. Models resulting from SKPSS tests performs better than those based on HEGY tests, except for DGP1 and DGP5 with $T=80$ or $T=120$. Forecast MSE often decreases with sample size for Model 1, Model 2 and Model 4, while the opposite is true for Model 3 and sometimes for Model 5 (not shown here). This is still valid when looking at the trace of the MSE matrix, but for both types of measurements it is within the Monte Carlo error bounds.

\section{Conclusions}

We propose a seasonal cointegration model for quarterly data which includes variables with different numbers of unit roots and thus need to be transformed in different ways to yield stationarity. A Monte Carlo simulation is carried out to investigate the consequences of specifying a SECM in annual differences in this situation. We compare the true model and the model where annually differenced variables are included to model specifications suggested by pre-tests for the numbers of unit roots. We consider two seasonal unit root tests in this analysis. One is the so called HEGY test, proposed by Hylleberg et al. (1990) and the other is the seasonal KPSS tests, proposed by Lyhagen (2000). We use seven different DGPs which all are based on estimates of quarterly observed income and consumption data for Japan, previously used in Engle et al. (1993). The True SECM, the SECM in annual differences and the two specifications suggested by the HEGY and the SKPSS tests are compared when the aim is to tests for cointegration. We consider tests for rank zero against 
full rank (rank two) and tests for rank one against full rank at all frequencies. Size and power properties are evaluated by estimating the proportion of rejections for each frequency. To evaluate the estimates of the parameters in the $\Pi$ matrices we use the mean of the mean squared error [MSE] for the same models. In the forecasting exercise we include a VAR model in annual differences. The results indicate that, in all practical cases where the true model is not known, a seasonal error correction model in annual differences may be a better choice, than relying on models which are specified according to seasonal unit root tests. This result holds true for both inference, as well as for estimation and forecasting. The second best choice when the true model is not known and when the aim is to forecast, is a VAR model in annual differences. These results extend those in Clements and Hendry (1997) where it was found that univariate models in annual differences may generate more accurate forecasts than models transformed according to HEGY test results.

\section{Acknowledgments}

Financial support for Johan Lyhagen from The Swedish Foundation for International Cooperation in Research and Higher Education (STINT) is gratefully acknowledged. Lyhagen is also grateful to Prof. M. H. Pesaran who acted as his sponsor at a Post-Doc stay with the Faculty of Economics and Politics, University of Cambridge.

\section{References}

Boswijk HP and Franses PH (1995) Periodic cointegration: Representation and inference. Review of Economics and Statistics 77: 436-454.

Clements MP and Hendry DF (1997) An empirical study of seasonal unit roots in forecasting. International Journal of Forecasting 13: 341-355.

Engle RF and Granger CWJ (1987) Co-integration and error correction: Representation, estimation and testing. Econometrica 55: 251-276.

Franses PH and Kunst R (1999) On the role of seasonal intercepts in seasonal cointegration. Oxford Bulletin of Economics and Statistics 61: 409-434.

Ghysels E, Lee HS and Noh J (1994) Testing for unit roots in seasonal time series: Some theoretical extensions and a Monte Carlo investigation. Journal of Econometrics 62: 415-442. 
Hylleberg S, Engle RF, Granger CWJ and Lee HS (1993) Seasonal integration and cointegration. Journal of Econometrics 44: 215-238.

Johansen S (1988) Statistical analysis of cointegration vectors. Journal of Economic Dynamics and Control 12, 231-254.

Johansen S (1991) Estimation and hypothesis testing of cointegration vectors in Gaussian vector autoregressive models. Econometrica 59: 1551-1580.

Johansen S (1995) Likelihood-based inference in cointegrated vector autoregressive models. Oxford university press, Oxford.

Johansen S and Schaumburg E (1998) Likelihood analysis of seasonal cointegration. Journal of Econometrics 88: 301-339.

Kunst R and Franses PH (1998) The impact of seasonal constants on forecasting seasonally cointegrated time series. Journal of Forecasting 17: 109-124.

Kwiatkowski D, Phillips PCB, Schmidt P and Shin Y (1992) Testing the null of stationarity against the alternative of a unit root: How sure are we that economic time series have a unit root? Journal of Econometrics 54: 159-178.

Löf M and Lyhagen J (1999) Forecasting performance of seasonal cointegration models. Working Paper Series in Economics and Finance, No 336, Stockholm School of Economics.

Lyhagen J (2000) The seasonal KPSS statistic. Working Paper Series in Economics and Finance, No 354, Stockholm School of Economics.

Maddala GS and Kim I-M (1998) Unit roots, Cointegration, and Structural Change. Cambridge University Press, Cambridge. 


\section{Appendix}

If we consider the seven cases presented in Section 4 the true filters for the second variable in the DGPs are:

$$
P(L)=\left[\begin{array}{r}
(1-L)(1+L)(1+i L)(1-i L) y_{2 t} \\
(1-L)(1+L) y_{2 t} \\
(1-L)(1-i L)(1+i L) y_{2 t} \\
(1+L)(1-i L)(1+i L) y_{2 t} \\
(1+L) y_{2 t} \\
(1-i L)(1+i L) y_{2 t} \\
(1-L) y_{2 t}
\end{array}\right]
$$

Note that $P(L)$ is not as defined in (9). Here it only concerns $y_{2 t}$ and the various filters are ordered after the DGP cases in (15). To test for cointegration at the zero and at the biannual frequency we use the following the true filters, for $y_{2 t}$, found from:

$$
Z_{m t}=\frac{P_{m}(L) L}{P_{m}\left(z_{m}\right) z_{m}} y_{2 t},
$$

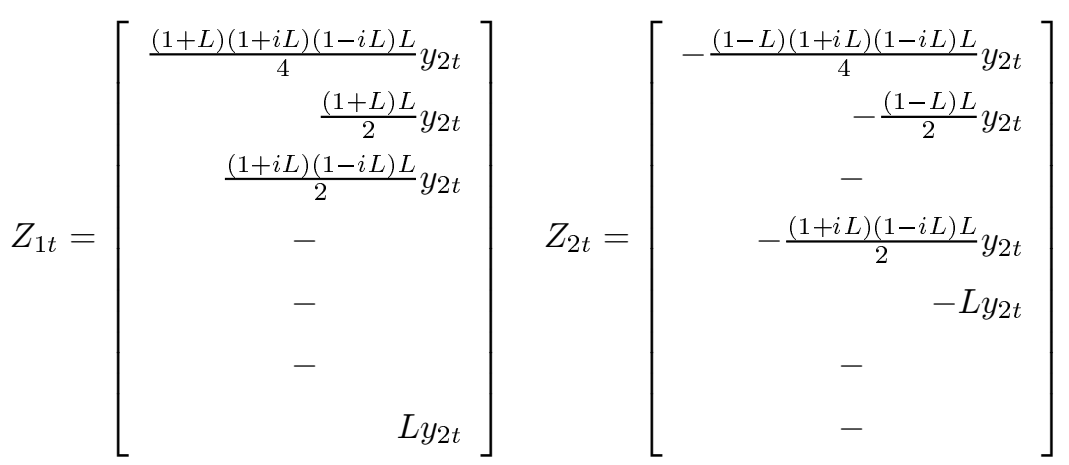

Finally, the true filters for variable two at the annual frequency are: 


$$
Z_{3 t}=\left[\begin{array}{c}
\frac{(1-L)(1+L)(1-i L) L}{4 i} y_{2 t} \\
- \\
\frac{(1-L)(1-i L) L}{2(i+1)} y_{2 t} \\
\frac{(1+L)(1-i L) L}{2(i-1)} y_{2 t} \\
- \\
\frac{(1-i L) L}{2 i} y_{2 t} \\
-
\end{array}\right] \quad Z_{4 t}=\left[\begin{array}{c}
-\frac{(1-L)(1+L)(1+i L) L}{4 i} y_{2 t} \\
- \\
-\frac{(1-L)(1+i L) L}{2(i-1)} y_{2 t} \\
-\frac{(1+L)(1+i L) L}{2(i+1)} y_{2 t} \\
- \\
-\frac{(1+i L) L}{2 i} y_{2 t} \\
-
\end{array}\right]
$$




\section{Tables}

Table 1: Roots inside the unit circle of the companion matrix.

\begin{tabular}{ccccccc}
\hline \hline & \multicolumn{7}{c}{ DGP } & & & \\
1 & 2 & 3 & 4 & 5 & 6 & 7 \\
\hline 0.92 & $0.23+0.22 \mathrm{i}$ & 0.65 & 0.53 & 0.96 & 0.97 & 0.25 \\
$-0.19+0.16 \mathrm{i}$ & $0.23-0.22 \mathrm{i}$ & 0.46 & $0.16+0.18 \mathrm{i}$ & 0 & 0.21 & 0 \\
$-0.19-0.16 \mathrm{i}$ & 0 & -0.09 & $0.16-0.18 \mathrm{i}$ & 0 & 0 & 0 \\
0.38 & 0 & 0 & 0 & 0 & 0 & 0 \\
\hline \hline
\end{tabular}

Table 2: Probability of rejecting $H_{0}: r=0$ at the zero frequency.

\begin{tabular}{ccccccccc}
\hline \hline & $\begin{array}{c}\text { DGP: } \\
\mathrm{T}\end{array}$ & {$[1]$} & {$[2]$} & {$[3]$} & 4 & 5 & 6 & {$[7]$} \\
\hline 40 & 1 & 0.58 & 0.82 & 0.98 & - & - & - & 0.97 \\
& 2 & 0.58 & 0.57 & 0.98 & $\underline{0.44}$ & $\underline{0.10}$ & $\underline{0.29}$ & 0.63 \\
& 3 & 0.53 & 0.71 & 0.93 & 0.09 & 0.05 & 0.34 & 0.04 \\
& 4 & 0.83 & 0.70 & 0.79 & 0.00 & 0.16 & 0.21 & 0.87 \\
\hline & & & & & & & & \\
\hline 80 & 1 & 0.90 & 1.00 & 1.00 & - & - & - & 1.00 \\
& 2 & 0.90 & 0.98 & 1.00 & $\underline{0.95}$ & $\underline{0.08}$ & $\underline{0.51}$ & 0.99 \\
& 3 & 0.84 & 0.82 & 0.95 & 0.12 & 0.04 & 0.57 & 0.00 \\
& 4 & 0.91 & 0.85 & 0.86 & 0.00 & 0.17 & 0.36 & 0.95 \\
\hline & & & & & & & & \\
\hline 120 & 1 & 0.99 & 1.00 & 1.00 & - & - & - & 1.00 \\
& 2 & 0.99 & 1.00 & 1.00 & $\underline{1.00}$ & $\underline{0.10}$ & $\underline{0.72}$ & 1.00 \\
& 3 & 0.90 & 0.79 & 0.95 & 0.14 & 0.04 & 0.77 & 0.00 \\
& 4 & 0.95 & 0.89 & 0.90 & 0.00 & 0.21 & 0.47 & 0.97 \\
\hline \hline
\end{tabular}

A unit root at the zero frequency exists for $y_{2 t}$ in DGP 1, 2, 3 and 7. These DGPs are within brackets. * A hyphon indicate that no $\Pi$ matrix exists for Model 1 at this frequency. Underlined numbers, for Model 2, indicate that $y_{2 t}$ is a stationary variable in the DGP at this frequency. See a summary of the various model specifications in Section 4. 
Table 3: Probability of rejecting $H_{0}: r=0$ at the biannual frequency.

\begin{tabular}{ccccccccc}
\hline \hline & $\begin{array}{c}\text { DGP: } \\
\text { Model }\end{array}$ & {$[1]$} & {$[2]$} & 3 & {$[4]$} & {$[5]$} & 6 & 7 \\
\hline 40 & 1 & 0.79 & 1.00 & - & 1.00 & 1.00 & - & - \\
& 2 & 0.79 & 0.76 & $\underline{1.00}$ & 0.97 & 0.86 & $\underline{1.00}$ & $\underline{0.60}$ \\
& 3 & 0.70 & 0.94 & 0.00 & 0.92 & 0.89 & 0.00 & 0.05 \\
& 4 & 0.11 & 0.66 & 0.00 & 0.39 & 0.52 & 0.00 & 0.00 \\
\hline & & & & & & & & \\
\hline 80 & 1 & 1.00 & 1.00 & - & 1.00 & 1.00 & - & - \\
& 2 & 1.00 & 1.00 & $\underline{1.00}$ & 1.00 & 1.00 & $\underline{1.00}$ & $\underline{0.99}$ \\
& 3 & 0.77 & 0.92 & 0.00 & 0.88 & 0.83 & 0.00 & 0.00 \\
& 4 & 0.20 & 0.75 & 0.00 & 0.52 & 0.65 & 0.00 & 0.00 \\
\hline & & & & & & & & \\
\hline 120 & 1 & 1.00 & 1.00 & - & 1.00 & 1.00 & - & - \\
& 2 & 1.00 & 1.00 & $\underline{1.00}$ & 1.00 & 1.00 & $\underline{1.00}$ & $\underline{1.00}$ \\
& 3 & 0.72 & 0.90 & 0.00 & 0.85 & 0.80 & 0.00 & 0.00 \\
& 4 & 0.25 & 0.80 & 0.00 & 0.59 & 0.73 & 0.00 & 0.00 \\
\hline \hline
\end{tabular}

A unit root at the biannual frequency exists for $y_{2 t}$ in DGP 1, 2, 4 and 5. These DGPs are within brackets. See ${ }^{*}$ in Table 2.

Table 4: Probability of rejecting $H_{0}: r=0$ at the annual frequency.

\begin{tabular}{ccccccccc}
\hline \hline & $\begin{array}{c}\text { DGP: } \\
\text { Model }\end{array}$ & {$[1]$} & 2 & {$[3]$} & {$[4]$} & 5 & {$[6]$} & 7 \\
\hline 40 & 1 & 1.00 & - & 1.00 & 1.00 & - & 1.00 & - \\
& 2 & 1.00 & $\underline{0.84}$ & 1.00 & 1.00 & $\underline{0.98}$ & 1.00 & $\underline{0.81}$ \\
& 3 & 0.92 & 0.01 & 0.88 & 0.83 & 0.01 & 0.88 & 0.10 \\
& 4 & 0.45 & 0.00 & 0.70 & 0.96 & 0.00 & 0.93 & 0.00 \\
\hline & & & & & & & & \\
\hline 80 & 1 & 1.00 & - & 1.00 & 1.00 & - & 1.00 & - \\
& 2 & 1.00 & 1.00 & 1.00 & 1.00 & $\underline{1.00}$ & 1.00 & $\underline{1.00}$ \\
& 3 & 0.88 & 0.01 & 0.90 & 0.79 & 0.03 & 0.85 & 0.15 \\
& 4 & 0.58 & 0.00 & 0.81 & 0.98 & 0.00 & 0.97 & 0.00 \\
\hline & & & & & & & & \\
\hline 120 & 1 & 1.00 & - & 1.00 & 1.00 & - & 1.00 & - \\
& 2 & 1.00 & $\underline{1.00}$ & 1.00 & 1.00 & $\underline{1.00}$ & 1.00 & $\underline{1.00}$ \\
& 3 & 0.85 & 0.01 & 0.92 & 0.78 & 0.05 & 0.81 & 0.18 \\
& 4 & 0.66 & 0.00 & 0.87 & 0.99 & 0.00 & 0.98 & 0.00 \\
\hline \hline
\end{tabular}

Unit roots at the annual frequency exists for $y_{2 t}$ in DGP 1, 3, 4 and 6.

These DGPs are within brackets. See ${ }^{*}$ in Table 2. 
Table 5: Probability of rejecting $H_{0}: r=1$ at the zero frequency.

\begin{tabular}{ccccccccc}
\hline \hline & $\begin{array}{c}\text { DGP: } \\
\text { Todel }\end{array}$ & {$[1]$} & {$[2]$} & {$[3]$} & 4 & 5 & 6 & {$[7]$} \\
\hline 40 & 1 & 0.12 & 0.06 & 0.10 & - & - & - & 0.06 \\
& 2 & 0.12 & 0.06 & 0.11 & 0.07 & 0.02 & 0.08 & 0.07 \\
& 3 & 0.08 & 0.04 & 0.10 & 0.02 & 0.01 & 0.08 & 0.00 \\
& 4 & 0.22 & 0.05 & 0.12 & 0.00 & 0.02 & 0.07 & 0.05 \\
\hline & & & & & & & & \\
\hline 80 & 1 & 0.10 & 0.06 & 0.08 & - & - & - & 0.06 \\
& 2 & 0.10 & 0.06 & 0.08 & 0.07 & 0.02 & 0.09 & 0.06 \\
& 3 & 0.07 & 0.02 & 0.08 & 0.02 & 0.01 & 0.09 & 0.00 \\
& 4 & 0.17 & 0.05 & 0.09 & 0.00 & 0.02 & 0.06 & 0.05 \\
\hline & & & & & & & & \\
\hline 120 & 1 & 0.09 & 0.06 & 0.07 & - & - & - & 0.06 \\
& 2 & 0.09 & 0.06 & 0.07 & 0.06 & 0.03 & 0.09 & 0.06 \\
& 3 & 0.08 & 0.02 & 0.07 & 0.03 & 0.01 & 0.10 & 0.00 \\
& 4 & 0.16 & 0.05 & 0.07 & 0.00 & 0.03 & 0.06 & 0.05 \\
\hline \hline
\end{tabular}

A unit root at the zero frequency exists for $y_{2 t}$ in DGP 1, 2, 3 and 7. These DGPs are within brackets. See ${ }^{*}$ in Table 2.

Table 6: Probability of rejecting $H_{0}: r=1$ at the biannual frequency.

\begin{tabular}{ccccccccc}
\hline \hline & $\begin{array}{c}\text { DGP: } \\
\mathrm{T}\end{array}$ & {$[1]$} & {$[2]$} & 3 & {$[4]$} & {$[5]$} & 6 & 7 \\
\hline 40 & 1 & 0.06 & 0.07 & - & 0.07 & 0.07 & - & - \\
& 2 & 0.06 & 0.07 & 0.07 & 0.07 & 0.07 & 0.08 & 0.07 \\
& 3 & 0.04 & 0.04 & 0.00 & 0.04 & 0.04 & 0.00 & 0.00 \\
& 4 & 0.00 & 0.03 & 0.00 & 0.02 & 0.03 & 0.00 & 0.00 \\
\hline & & & & & & & & \\
\hline 80 & 1 & 0.06 & 0.06 & - & 0.07 & 0.06 & - & - \\
& 2 & 0.06 & 0.06 & 0.07 & 0.07 & 0.07 & 0.07 & 0.06 \\
& 3 & 0.03 & 0.02 & 0.00 & 0.03 & 0.02 & 0.00 & 0.00 \\
& 4 & 0.01 & 0.03 & 0.00 & 0.02 & 0.03 & 0.00 & 0.00 \\
\hline & & & & & & & & \\
\hline 120 & 1 & 0.06 & 0.06 & - & 0.06 & 0.06 & - & - \\
& 2 & 0.06 & 0.06 & 0.06 & 0.06 & 0.06 & 0.06 & 0.06 \\
& 3 & 0.04 & 0.02 & 0.00 & 0.02 & 0.02 & 0.00 & 0.00 \\
& 4 & 0.01 & 0.03 & 0.00 & 0.02 & 0.02 & 0.00 & 0.00 \\
\hline \hline
\end{tabular}

A unit root at the biannual zero frequency exists for $y_{2 t}$ in DGP 1, 2, 4 and 5. These DGPs are within brackets. See ${ }^{*}$ in Table 2. 
Table 7: Probability of rejecting $H_{0}: r=1$ at the annual frequency.

\begin{tabular}{ccccccccc}
\hline \hline & $\begin{array}{c}\text { DGP: } \\
\mathrm{T}\end{array}$ & {$[1]$} & 2 & {$[3]$} & {$[4]$} & 5 & {$[6]$} & 7 \\
\hline 40 & 1 & 0.07 & - & 0.07 & 0.07 & - & 0.07 & - \\
& 2 & 0.07 & 0.06 & 0.07 & 0.07 & 0.07 & 0.07 & 0.06 \\
& 3 & 0.05 & 0.00 & 0.03 & 0.01 & 0.00 & 0.03 & 0.01 \\
& 4 & 0.03 & 0.00 & 0.05 & 0.04 & 0.00 & 0.06 & 0.00 \\
\hline & & & & & & & & \\
\hline 80 & 1 & 0.06 & - & 0.06 & 0.06 & - & 0.05 & - \\
& 2 & 0.06 & 0.06 & 0.06 & 0.06 & 0.06 & 0.06 & 0.05 \\
& 3 & 0.07 & 0.00 & 0.02 & 0.01 & 0.00 & 0.02 & 0.01 \\
& 4 & 0.09 & 0.00 & 0.06 & 0.05 & 0.00 & 0.05 & 0.00 \\
\hline & & & & & & & & \\
\hline 120 & 1 & 0.06 & - & 0.06 & 0.05 & - & 0.05 & - \\
& 2 & 0.06 & 0.05 & 0.05 & 0.05 & 0.05 & 0.06 & 0.05 \\
& 3 & 0.10 & 0.00 & 0.02 & 0.01 & 0.00 & 0.02 & 0.01 \\
& 4 & 0.15 & 0.00 & 0.06 & 0.05 & 0.00 & 0.05 & 0.00 \\
\hline \hline
\end{tabular}

Unit roots at the annual frequency exists for $y_{2 t}$ in DGP $1,3,4$ and 6. These DGPs are within brackets. See * in Table 2.

Table 8: Mean of MSE, $\Pi_{1}$.

\begin{tabular}{ccccccccc}
\hline \hline & $\begin{array}{c}\text { DGP: } \\
\mathrm{T}\end{array}$ & {$[1]$} & {$[2]$} & {$[3]$} & 4 & 5 & 6 & {$[7]$} \\
\hline 40 & 1 & 0.06 & 0.07 & 0.02 & - & - & - & 0.04 \\
& 2 & 0.06 & 0.12 & 0.03 & $\underline{0.47}$ & $\underline{0.06}$ & $\underline{0.02}$ & 0.11 \\
& 3 & 0.18 & 0.11 & 0.04 & 0.04 & 0.05 & 0.04 & 0.33 \\
& 4 & 1.04 & 0.22 & 0.09 & 0.00 & 0.15 & 0.02 & 0.07 \\
\hline & & & & & & & & \\
\hline 80 & 1 & 0.01 & 0.03 & 0.01 & - & - & - & 0.02 \\
& 2 & 0.01 & 0.04 & 0.01 & $\underline{0.37}$ & $\underline{0.01}$ & $\underline{0.01}$ & 0.04 \\
& 3 & 0.22 & 0.09 & 0.03 & 0.03 & 0.01 & 0.03 & 0.35 \\
& 4 & 1.03 & 0.15 & 0.05 & 0.00 & 0.03 & 0.00 & 0.03 \\
\hline & & & & & & & & \\
\hline 120 & 1 & 0.01 & 0.02 & 0.01 & - & - & - & 0.01 \\
& 2 & 0.01 & 0.03 & 0.01 & $\underline{0.34}$ & $\underline{0.01}$ & $\underline{0.00}$ & 0.02 \\
& 3 & 0.30 & 0.09 & 0.02 & 0.04 & 0.00 & 0.04 & 0.35 \\
& 4 & 1.01 & 0.12 & 0.04 & 0.00 & 0.02 & 0.00 & 0.02 \\
\hline \hline
\end{tabular}

A $\Pi_{1}$ matrix does not not exists for Model 1 when DGP 4, 5 and 6 are used. Other DGPs are within brackets. See * in Table 2. 
Table 9: Mean of MSE, $\Pi_{2}$.

\begin{tabular}{ccccccccc}
\hline \hline & $\begin{array}{c}\text { DGP: } \\
\mathrm{T}\end{array}$ & {$[1]$} & {$[2]$} & 3 & {$[4]$} & {$[5]$} & 6 & 7 \\
\hline 40 & 1 & 0.15 & 0.09 & - & 0.08 & 0.01 & - & - \\
& 2 & 0.15 & 0.22 & $\underline{2.15}$ & 0.27 & 0.20 & $\underline{1.67}$ & $\underline{0.60}$ \\
& 3 & 0.84 & 0.17 & 0.00 & 0.37 & 0.22 & 0.00 & 0.02 \\
& 4 & 1.03 & 0.53 & 0.00 & 1.67 & 0.71 & 0.00 & 0.00 \\
\hline & & & & & & & & \\
\hline 80 & 1 & 0.06 & 0.04 & - & 0.04 & 0.00 & - & - \\
& 2 & 0.06 & 0.09 & $\underline{2.09}$ & 0.11 & 0.08 & $\underline{1.63}$ & $\underline{0.50}$ \\
& 3 & 0.70 & 0.16 & 0.00 & 0.43 & 0.27 & 0.00 & 0.00 \\
& 4 & 0.95 & 0.38 & 0.00 & 1.31 & 0.51 & 0.00 & 0.00 \\
\hline & & & & & & & & \\
\hline 120 & 1 & 0.04 & 0.02 & - & 0.02 & 0.00 & - & - \\
& 2 & 0.04 & 0.06 & $\underline{2.06}$ & 0.07 & 0.05 & $\underline{1.62}$ & $\underline{0.46}$ \\
& 3 & 0.66 & 0.17 & 0.00 & 0.48 & 0.29 & 0.00 & 0.00 \\
& 4 & 0.87 & 0.30 & 0.00 & 1.11 & 0.40 & 0.00 & 0.00 \\
\hline \hline
\end{tabular}

$\overline{\mathrm{A} \Pi_{2} \text { matrix does not not exists for Model } 1 \text { when DGP 3, } 6 \text { and } 7 \text { are }}$ used. Other DGPs are within brackets. See ${ }^{*}$ in Table 2.

Table 10: Mean of MSE, $\Pi_{3}$.

\begin{tabular}{ccccccccc}
\hline \hline & $\begin{array}{c}\text { DGP: } \\
\mathrm{T}\end{array}$ & {$[1]$} & 2 & {$[3]$} & {$[4]$} & 5 & $6]$ & 7 \\
\hline 40 & 1 & 0.07 & - & 0.04 & 0.07 & - & 0.18 & - \\
& 2 & 0.07 & $\underline{0.29}$ & 0.07 & 0.08 & $\underline{0.31}$ & 0.30 & $\underline{0.32}$ \\
& 3 & 0.36 & 0.00 & 0.16 & 0.24 & 0.00 & 0.28 & 0.03 \\
& 4 & 1.65 & 0.00 & 0.35 & 0.28 & 0.00 & 0.24 & 0.00 \\
\hline 80 & 1 & 0.03 & - & 0.02 & 0.03 & - & 0.08 & - \\
& 2 & 0.03 & $\underline{0.26}$ & 0.03 & 0.03 & $\underline{0.28}$ & 0.12 & $\underline{0.28}$ \\
& 3 & 0.49 & 0.01 & 0.12 & 0.25 & 0.02 & 0.19 & 0.05 \\
& 4 & 1.82 & 0.00 & 0.21 & 0.18 & 0.00 & 0.10 & 0.00 \\
\hline 120 & 1 & 0.02 & - & 0.01 & 0.02 & - & 0.05 & - \\
& 2 & 0.02 & $\underline{0.25}$ & 0.02 & 0.02 & $\underline{0.27}$ & 0.08 & $\underline{0.27}$ \\
& 3 & 0.68 & 0.01 & 0.09 & 0.26 & 0.03 & 0.19 & 0.07 \\
& 4 & 1.99 & 0.00 & 0.15 & 0.15 & 0.00 & 0.06 & 0.00 \\
\hline \hline
\end{tabular}

$\mathrm{A} \Pi_{3}$ matrix does not not exists for Model 1 when DGP 2, 5 and 7 are used. Other DGPs are within brackets. See ${ }^{*}$ in Table 2. 
Table 11: Mean of MSE, $\Pi_{4}$.

\begin{tabular}{cccccccccc}
\hline \hline & $\begin{array}{c}\text { DGP: } \\
\mathrm{T}\end{array}$ & {$[1]$} & 2 & {$[3]$} & {$[4]$} & 5 & {$[6]$} & 7 \\
\hline 40 & 1 & 0.06 & - & 0.06 & 0.07 & - & 0.02 & - \\
& 2 & 0.06 & $\underline{0.07}$ & 0.06 & $\underline{0.09}$ & 0.24 & 0.10 & $\underline{0.05}$ \\
& 3 & 0.11 & 0.00 & 0.18 & 0.14 & 0.00 & 0.45 & 0.01 \\
& 4 & 0.71 & 0.00 & 1.27 & 0.51 & 0.00 & 0.26 & 0.00 \\
\hline & & & & & & & & \\
\hline 80 & 1 & 0.03 & - & 0.03 & 0.03 & - & 0.01 & - \\
& 2 & 0.03 & $\underline{0.05}$ & 0.03 & $\underline{0.04}$ & 0.23 & 0.04 & $\underline{0.03}$ \\
& 3 & 0.09 & 0.00 & 0.12 & 0.12 & 0.00 & 0.50 & 0.01 \\
& 4 & 0.52 & 0.00 & 0.96 & 0.38 & 0.00 & 0.12 & 0.00 \\
\hline & & & & & & & & \\
\hline 120 & 1 & 0.02 & - & 0.02 & 0.02 & - & 0.01 & - \\
& 2 & 0.02 & $\underline{0.05}$ & 0.02 & $\underline{0.02}$ & 0.23 & 0.03 & $\underline{0.03}$ \\
& 3 & 0.10 & 0.00 & 0.09 & 0.12 & 0.00 & 0.62 & 0.01 \\
& 4 & 0.37 & 0.00 & 0.72 & 0.32 & 0.00 & 0.07 & 0.00 \\
\hline \hline
\end{tabular}

A $\Pi_{4}$ matrix does not not exists for Model 1 when DGP 2, 5 and 7 are used. Other DGPs are within brackets. See ${ }^{*}$ in Table 2.

Table 12: Ranking of forecasting performance (average over 12 periods). The measure is the determinant of the MSE marix.

\begin{tabular}{c|ccccc|ccccc|ccccc}
\hline \\
Mod. & 1 & 2 & 3 & 4 & 5 & 1 & 2 & 3 & 4 & 5 & 1 & 2 & 3 & 4 & 5 \\
\hline DGP & & & & & & & & & & & & & & \\
1 & 1.3 & 1.3 & 3.9 & 5.0 & 2.6 & 1.0 & 1.0 & 4.0 & 5.0 & 3.0 & 1.0 & 1.0 & 4.5 & 4.5 & 3.0 \\
2 & 1.0 & 2.0 & 3.6 & 4.1 & 4.3 & 1.0 & 2.0 & 5.0 & 3.3 & 3.8 & 1.0 & 2.0 & 5.0 & 3.3 & 3.8 \\
3 & 1.0 & 2.0 & 4.9 & 4.1 & 3.0 & 1.0 & 2.0 & 5.0 & 4.0 & 3.0 & 1.0 & 2.0 & 5.0 & 4.0 & 3.0 \\
4 & 1.3 & 2.5 & 5.0 & 3.7 & 2.5 & 1.3 & 2.3 & 5.0 & 3.7 & 2.8 & 1.1 & 2.3 & 5.0 & 3.7 & 3.0 \\
5 & 1.3 & 3.9 & 3.2 & 4.4 & 2.2 & 1.0 & 2.6 & 3.9 & 4.5 & 3.0 & 1.0 & 2.3 & 3.9 & 4.1 & 3.8 \\
6 & 1.5 & 3.0 & 5.0 & 3.4 & 2.1 & 1.0 & 2.0 & 5.0 & 3.3 & 3.7 & 1.0 & 2.0 & 5.0 & 3.2 & 3.0 \\
7 & 1.0 & 3.0 & 5.0 & 2.0 & 4.0 & 1.0 & 2.1 & 5.0 & 2.9 & 4.0 & 1.0 & 2.0 & 5.0 & 3.0 & 4.0 \\
\end{tabular}

